\title{
NON-HYDROSTATIC TRANSITIONAL OPEN-CHANNEL FLOWS FROM A SUPERCRITICAL TO A SUBCRITICAL STATE
}

Yebegaeshet T. ZERIHUN ${ }^{1 *}$

\section{Abstract}

In this study, a depth-averaged numerical model was employed to investigate the two-dimensional flow features of transitional open-channel flows from a supercritical to a subcritical state. Compared to a shallow-water model, the proposed model incorporates supplementary terms to account for the effects of non-uniform velocity and non-hydrostatic pressure distributions. The model equation was solved numerically by means of the Adams-Bashforth-Moulton scheme. A wide variety of transitional open-channel flow problems such as hydraulic jumps was considered for assessing the suitability of the numerical model. The results of the model for the free-surface profile, pressure distribution, and characteristics of the first wave of an undular jump were compared with the experimental data, and the agreement was found to be satisfactory. Despite the effects of the three-dimensional characteristics of the flow and the bulking of the flow caused by air entrainment, the model performed reasonably well with respect to the simulations of the mean flow characteristics of the curvilinear turbulent flow problems. Furthermore, the results of this investigation confirmed that the model is more suitable for analyzing near-critical turbulent flow problems without cross-channel shock waves.

\section{Address}

1 David \& James - Engineering and Environmental Consultancy, 204 Albion Road, Victoria 3350, Australia

\section{* Corresponding author: zyebegaeshet@gmail.com}

\section{Key words}

- Undular open-channel flow,

- Hydraulic jump

- Near-critical turbulent flow,

- Flow instability,

- Numerical modeling,

- Curvilinear transitional flow.

\section{INTRODUCTION}

A hydraulic jump occurs whenever a flow changes from a supercritical to a subcritical state and in the process passes through rapid changes in its free-surface elevation. This virulent phenomenon is characterized by the prevalence of high turbulence, air entrainment, surface waves, and a non-hydrostatic pressure distribution that invalidates the application of the shallow-water theory. The hydraulic characteristics of the jump are affected by the tailwater depth; a free jump or a submerged jump can be formed, or a two-dimensional (2D) undular hydraulic jump may be produced, depending on the Froude number of the approach flow. A comprehensive investigation of the three types of jumps will be the task of this study.
Bélanger (1845) was the first to perform a theoretical analysis of a free hydraulic jump by applying the conservation laws of mass and momentum. Almost a century later, Bakhmeteff and Matzke (1936) conducted a detailed experimental study and developed a curve that correlates the normalized length of this jump to the kinetic flow factor. After analyzing a large set of experimental data, Rajaratnam and Subramanya (1968) and Hager (1993) developed empirical equations for the free-surface profile of a rectangular hydraulic jump. However, the overall accuracy and application range of such types of empirical results rely on the scope of the experiments. Others have experimentally investigated the characteristics of the turbulence of the free hydraulic jump, including the Reynolds stresses, the bubbly two-phase flow regime, and the structure of the flow (e.g., Resch and Leutheusser, 
1972a,b; Resch et al., 1974). Liu et al. (2004) and Mignot and Cienfuegos (2010) have also made important contributions to the understanding of the characteristics of turbulence. As an alternative to the experimental methods, a classical numerical approach based on shallow-water equations has been used to analyze the hydraulic jump problems (e.g., Madsen et al., 2005; Richard and Gavrilyuk, 2013). This approach treats the jump as a thin shock with a dissipative shock-capturing scheme and gives reasonably good results for the jump locations. However, the shape and length of the jump have not been predicted satisfactorily by this method. To overcome such drawbacks, higher-order modeling approaches have been used to obtain a greater insight into the structure of the free hydraulic jump (e.g., McCorquodale and Khalifa, 1983; Qingchao and Drewes, 1994; Khan and Steffler, 1996; Bayon-Barrachina and Lopez-Jimenez, 2015; Witt et al., 2018).

The flow fields of the submerged hydraulic jump have been experimentally studied by various investigators. Govinda Rao and Rajaratnam (1963) studied this jump and obtained empirical equations for the prominent flow parameters. In addition to the macroscopic features, other mean and turbulent flow characteristics of the jump have been investigated by Long (1991), Dey and Sarkar (2008), and Padulano et al. (2017). Alternatively, Long et al. (1991) and Ma et al. (2001) applied higher-order numerical models based on the Reynolds-averaged Navier-Stokes (RANS) equations to simulate the mean flow characteristics of a submerged hydraulic jump in open channels. However, the offset control volume method used by Long et al. (1991) for the treatment of the free surface is applicable only to open-channel flows with small free-surface slopes and negligible vertical velocity.

As in the cases of free and submerged hydraulic jumps, the undular hydraulic jump has been thoroughly investigated. Recent experimental studies, which detailed the characteristics of the undular flow, include Ryabenko (1990), Chanson (1995, 2000), Chanson and Montes (1995), Reinauer and Hager (1995), and Ohtsu et al. (2001). Based on the upper limit of the approaching Froude number and the coefficient of the degree of non-hydrostatics, Ryabenko (1990) suggested a more precise definition of the conditions of the existence of an undular hydraulic jump. Montes (1986) aptly discussed the significance of boundary friction in modeling this jump using a higher-order energy equation. More recently, Chanson (2009) presented a comprehensive review of previous experimental investigations and emphasized the limited knowledge of the internal structure of the hydraulic jumps. Using the potential-flow theory, the problems of the undular hydraulic jump were studied numerically by treating part of the first wave crest as a solitary wave, including its transition from a supercritical to a subcritical state, and/or the downstream subcritical flow regime as a series of cnoidal waves (e.g., Fawer, 1937; Benjamin and Lighthill, 1954; Mandrup-Andersen, 1978; Hager and Hutter, 1984). By applying the logarithmic law of the wall, Grillhofer and Schneider (2003) presented an asymptotic approach for simplifying the RANS equations and obtained an ordinary differential equation describing the free-surface profile. In addition, a Boussinesq approximation was employed by Abbott and Rodenhuis (1972), Montes and Chanson (1998), and Bose et al. (2012) for the solutions of the undular jump problems. However, the applicability of the approaches of Abbott and Rodenhuis (1972) and Bose et al. (2012) is restricted to open-channel flows with slightly sloped and curved streamlines.

The current study therefore has two primary objectives. The first objective is to develop an alternative depth-averaged numerical model for simulating the internal flow structure of the aforementioned three types of hydraulic jumps. The second is to thoroughly examine the suitability of the proposed model for such types of transitional open-channel flow problems. The approach presented here accounts for the effects of non-uniform horizontal velocity and non-hydrostatic pressure distributions, thereby overcoming the limitations of the shallow-water and the lower-order Boussinesq-type equations. By ignor- ing the variations of the flow parameters in the transverse direction, such a higher-order approach simplifies the three-dimensional (3D) turbulent open-channel flow problem into a $2 \mathrm{D}$ problem on a vertical plane. Consequently, the model provides an approximate description of the salient features of the curvilinear transitional flow. This modeling technique enables us to better understand the linkage of the wavelength and amplitude of an undular flow with a streamwise momentum balance. Compared to a more detailed 3D numerical model, the proposed model is computationally efficient and is relatively simple to apply, making it a preferable design tool for developing or modifying the conceptual design of an energy dissipator. It is also a part of this study to critically assess the validity of the numerical results by comparing the model solutions with the available experimental data in the literature. It is worth noting that the effects of air entrainment in the rapidly-varied turbulent open-channel flows are ignored in this work.

Following a brief summary of the derivation of the governing equations, the paper presents a detailed description of the application of the Adams-Bashforth-Moulton numerical scheme for the solutions of the flow equation. The initial and boundary conditions of the hydraulic jump problems are also discussed. A brief discussion of the results of the model is presented by comparing the predictions to the experimental data from the literature. The paper is ended by summarizing the main findings of the investigation.

\section{FORMULATION OF EQUATIONS}

Under consideration is a moderately curved open-channel flow in a rigid-bed rectangular channel. The Cartesian coordinates are chosen in such a way that $x$ is horizontally along the channel [L]; $y$ is horizontally in the transverse direction [L]; and $z$ is vertically upward [L], as illustrated in Fig. 1. By considering the momentum balance in the streamwise $x$-direction, Fenton and Zerihun (2007) presented a dynamic equation, which is given by

$$
\frac{\partial Q}{\partial t}+\beta \frac{\partial}{\partial x}\left(\frac{Q^{2}}{A}\right)+\frac{1}{\rho} \int_{A} \frac{\partial p}{\partial x} d A+\frac{f Q^{2} P_{w}}{8 A^{2}}\left(1+\zeta_{x}^{2}\right)=0
$$

where $f$ (dimensionless) denotes the Darcy-Weisbach friction coefficient; $Q$ is the discharge $\left[\mathrm{L}^{3} \mathrm{~T}^{-1}\right] ; A$ is the cross-sectional area of the flow $\left[\mathrm{L}^{2}\right] ; \rho$ is the density of the fluid $\left[\mathrm{ML}^{-3}\right] ; p$ is the pressure $[\mathrm{ML}-$ $\left.{ }^{1} \mathrm{~T}^{-2}\right] ; \beta$ (dimensionless) refers to the Boussinesq momentum coefficient; $P_{w}$ is the wetted perimeter $[\mathrm{L}] ; \zeta_{x}$ (dimensionless) is the local bed gradient of the channel; and $t$ is the time [T].

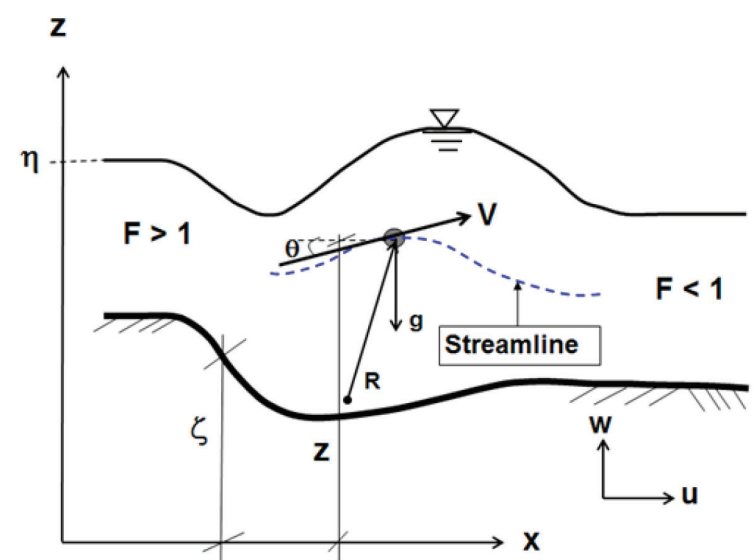

Fig. 1 Definition sketch of a transitional open-channel flow over a curved bed. The velocity tangent to the streamline and the Froude number are denoted by $V$ and $F$, respectively 
The Euler equation for the momentum balance in the vertical direction can be written as

$$
\frac{1}{\rho} \frac{\partial p}{\partial z}=-g-a_{z}
$$

where $g$ is the gravitational acceleration $\left[\mathrm{LT}^{-2}\right]$, and $a_{z}$ is the vertical acceleration of the flow $\left[\mathrm{LT}^{-2}\right]$. For a non-hydrostatic open-channel flow problem, the rates of change of quantities over time are much slower than the apparent acceleration of a fluid particle following a curvilinear path (Fenton and Zerihun, 2007). Using this approximation, the expression for the vertical acceleration becomes

$$
a_{z}=u \frac{\partial w}{\partial x}+w \frac{\partial w}{\partial z}=\kappa \frac{u^{2}}{\cos ^{3} \theta}=u^{2} \xi,
$$

where $\kappa(=1 / R)$ is the curvature of the streamline $\left[\mathrm{L}^{-1}\right] ; R$ is the radius of curvature [L]; $u$ is the horizontal velocity $\left[\mathrm{LT}^{-1}\right] ; w$ is the vertical velocity $\left[\mathrm{LT}^{-1}\right]$; and $\theta$ is the angle of inclination of the streamline with the horizontal axis [deg] (see Fig. 1). The above equation requires a closure hypothesis for the vertical distribution of the streamline geometry parameter $\xi$. For simplicity, a linear profile is assumed as in Zerihun (2008, 2017a),

$$
\begin{gathered}
\xi=\xi_{b}+\left(\xi_{s}-\xi_{b}\right) \lambda, \\
\lambda=\frac{z-\zeta}{\eta-\zeta},
\end{gathered}
$$

where $\eta$ and $\zeta$ are the elevations of the free surface and channel bed $[\mathrm{L}]$, respectively; $\lambda$ is a non-dimensional vertical height; and $z$ is the vertical coordinate of a point in the flow field $[\mathrm{L}]$. The subscripts $b$ and $s$ refer to the magnitudes of the parameter at the channel bed and free surface, respectively. The variation of the horizontal velocity across the flow depth is also given by a linear relation as follows:

$$
\begin{aligned}
& u=\frac{q}{H} G(\omega, \lambda)=\frac{q}{H}(2 \lambda(1-\omega)+\omega), \\
& \beta=\frac{1}{U^{2}} \int_{0}^{1} u^{2}(\lambda) d \lambda=\frac{1}{3}\left(\omega^{2}-2 \omega+4\right),
\end{aligned}
$$

where $G(\omega, \lambda)$ determines the shape of the distribution of the horizontal component of the velocity and satisfies the condition $\int G(\omega, \lambda) d \lambda=1$ for recovering the depth-averaged velocity $U=(q / H) ; H$ is the depth of flow [L]; $\omega$ (dimensionless) is the velocity distribution parameter; and $q$ is the discharge per unit width $\left[\mathrm{L}^{2} \mathrm{~T}^{-1}\right]$.

Based on Leibnitz's rule, the integration of the continuity equation for a $2 \mathrm{D}$ flow can be written as

$$
\begin{aligned}
& \int_{z}^{\eta} \frac{\partial u}{\partial x} d z+\int_{z}^{\eta} \frac{\partial w}{\partial z} d z=\frac{\partial}{\partial x}\left(\int_{z}^{\eta} u(z) d z\right)+u(z) z_{x}-u(\eta) \eta_{x} \\
& +w_{S}-w=0
\end{aligned}
$$

where the subscript $x$ denotes a partial differentiation with respect to the horizontal axis, and $u_{s}$ and $w_{s}$ are the free-surface velocity components in the horizontal and vertical directions $\left[\mathrm{LT}^{-1}\right]$, respectively.

After substituting Eq. (6a) into Eq. (7), the first term on the right-hand side of Eq. (7) is integrated and then differentiated with respect to $x$. Using the free-surface kinematic boundary condition, $w_{S}=\partial H / \partial t+(\partial \eta / \partial x) u_{s}$, the resulting expression is simplified to give the following equation:

$$
\begin{aligned}
& w=\frac{\partial H}{\partial t}\left(1-\omega(1-\lambda)-(1-\omega)\left(1-\lambda^{2}\right)\right)+\frac{q \eta_{x}}{H}(2-\omega) \\
& -\frac{2 q \zeta_{x}}{H}((1-\omega)(1-\lambda))-\frac{q H_{x}}{H}((1-\lambda) \omega) \\
& -\frac{2 q H_{x}}{H}\left((1-\omega)\left(1-\lambda^{2}\right)\right)
\end{aligned}
$$

Inserting Eqs. (4) and (6a) into Eq. (2) and then vertically integrating the resulting expression from $z$ to $\eta$ leads to the pressure distribution equation for flow in a rectangular channel

$$
\begin{aligned}
& \frac{p}{\rho g}=H(1-\lambda)+\frac{Q^{2}}{g H B^{2}}\left(\zeta_{x x} \omega^{2}(1-\lambda)+\left(\frac{H_{x x} \omega^{2}}{2}\right)\left(1-\lambda^{2}\right)\right) \\
& +\frac{Q^{2}}{g H B^{2}}\left(2 \zeta_{x x} \omega(1-\omega)\left(1-\lambda^{2}\right)\right)+\frac{Q^{2}}{g H B^{2}}\left(\left(\zeta_{x x}(1-\omega)^{2}\right) \frac{4\left(1-\lambda^{3}\right)}{3}\right) \\
& +\frac{Q^{2}}{g H B^{2}}\left(\left(H_{x x} \omega(1-\omega)\right) \frac{4\left(1-\lambda^{3}\right)}{3}+H_{x x}(1-\omega)^{2}\left(1-\lambda^{4}\right)\right),
\end{aligned}
$$

where $B$ is the width of the channel [L], and $\zeta_{x x}$ is the second derivative of the bed profile $\left[\mathrm{L}^{-1}\right]$. The above equation includes a higher-order dynamic pressure correction that stems from terms accounting for the effects of the vertical curvatures of the streamline and a non-uniform horizontal velocity distribution. If one considers a moderately curved flow with a pre-assumed uniform horizontal velocity distribution $\left(\omega=1+\eta_{x}^{2}=1+\zeta_{x}^{2} \cong 1.0\right)$, then the pressure equation of Zerihun $(2008,2017 \mathrm{~b})$ will be retrieved.

Ignoring the variation of the free-surface elevation in the transverse direction, Eq. (9) is differentiated with respect to $x$ and then integrated across the channel. After substituting the resulting expression into Eq. (1) and rearranging similar terms together, the governing equation for a curvilinear open-channel flow is obtained as follows:

$$
\begin{gathered}
\frac{\partial Q}{\partial t}+\left(\beta \frac{2}{H}+\frac{\sigma_{1}}{3} \zeta_{x x}+\frac{2 \sigma_{2}}{15} H_{x x}\right) \frac{Q}{B} \frac{\partial Q}{\partial x}+\frac{\sigma_{2}}{15} \frac{Q^{2}}{B} H_{x x x} \\
+\frac{\sigma_{1}}{6} \frac{Q^{2}}{A} \zeta_{x} H_{x x}+\left(g A-\beta \frac{Q^{2} B}{A^{2}}\right) H_{x} \\
+\frac{Q^{2}}{A}\left(\frac{\sigma_{1}}{6} \zeta_{x x x} H+\frac{\sigma_{3}}{3} \zeta_{x x} \zeta_{x}\right)+g A \zeta_{x}+\frac{f Q^{2} P_{w}}{8 A^{2}}\left(1+\zeta_{x}^{2}\right)=0, \\
\sigma_{1}=\omega^{2}-4 \omega+6, \\
\sigma_{2}=2 \omega^{2}-9 \omega+12, \\
\sigma_{3}=\omega^{2}-2 \omega+4,
\end{gathered}
$$

where $\zeta_{x x x}$ is the third derivative of the bed profile $\left[\mathrm{L}^{-2}\right]$. It is apparent from Eq. (10a) that the main differences between this Boussinesq-type equation and earlier equations presented by Fenton and Zerihun (2007) and Zerihun $(2008,2016)$ are all in the non-linear coefficients associated with the spatial derivative terms. In Eq. (10a), these coefficients take into account the effect of a non-uniform horizontal velocity distribution in addition to the effect of the vertical acceleration of the flow. In this study, the flow resistance due to the shear forces of the boundary is estimated by using the Darcy-Weisbach equation with an explicit form of the Colebrook-White formula (Zigrang and Sylvester, 1982) for the friction factor. It is important to note that the effects of the turbulence characteristics of the flow were ignored in the development of the above equations. For a case 
of no lateral inflow or outflow from the channel, the depth-averaged continuity equation is given by

$$
B \frac{\partial H}{\partial t}+\frac{\partial Q}{\partial x}=0 .
$$

The above equation is numerically coupled with Eq. (10a) for the complete description of the unsteady, transitional open-channel flows with a predominant effect of non-hydrostatic pressure distribution. In the next section, the derivation of the approximate analytical expressions for the first wave characteristics of an undular jump will be presented.

\subsection{Solution of the linearized equation}

For a steady undular flow in a constant slope channel $\left(\zeta_{x x}=\zeta_{x x x}=0\right)$, Eq. $(10 a)$ is reduced to

$$
\begin{aligned}
& H_{x x x}-\frac{5 \sigma_{1} S_{0}}{2 H \sigma_{2}} H_{x x}+\left(\frac{15 g H}{q^{2} \sigma_{2}}-\beta \frac{15}{H^{2} \sigma_{2}}\right) H_{x} \\
& +\frac{15 g H}{q^{2} \sigma_{2}}\left(S_{f}-S_{0}\right)=0,
\end{aligned}
$$

where $S_{0}$ (dimensionless) is the slope of the channel bed, and $S_{f}$ (dimensionless) is the friction slope. Following Boussinesq's (1877) approach, the above equation is linearized by assuming that the deviation of the perturbation depth, $h$, from the subcritical sequent depth, $D$, is small (see Fig. 2). Based on this approximation, the depth of the undular flow is expressed as:

$$
H=D+h \text {. }
$$

Substituting Eq. (13) into Eq. (12), and ignoring the products of any two small terms (i.e., $h h_{x} / D$ ), yields a third-order linear differential equation to the first-order of approximation as follows:

$$
\begin{gathered}
h_{x x x}+\frac{\Gamma_{1}}{D} h_{x x}+\frac{\Gamma_{2}}{D^{2}} h_{x}+\frac{\Gamma_{3}}{D^{3}} h=0, \\
\Gamma_{1}=-\frac{5 \sigma_{1} S_{0}}{2 \sigma_{2}}, \\
\Gamma_{2}=\frac{15}{F_{m}{ }^{2} \sigma_{2}}-\beta \frac{15}{\sigma_{2}}, \\
\Gamma_{3}=-\frac{50 S_{0}}{F_{m}{ }^{2} \sigma_{2}} .
\end{gathered}
$$

A sinusoidal perturbation depth can be expressed as the real part of

$$
h(x)=C_{1} \exp \left(-N x+i k\left(x-C_{2}\right)\right),
$$

where $C_{1}$ and $C_{2}$ are constants [L], which can be determined from the boundary conditions; $N$ is the positive root of the characteristic equation $\left[\mathrm{L}^{-1}\right] ; k$ is the wave number $\left[\mathrm{L}^{-1}\right]$; and $i$ (dimensionless) is the imaginary unit. In order to obtain the expressions involving $N$ and $k$, Eq. (15) and its first, second, and third derivatives are substituted into Eq. (14a). Separating the real and imaginary parts of the resulting expression leads to

$$
\begin{gathered}
k^{2}=3 N^{2}-\frac{2 \Gamma_{1}}{D} N+\frac{\Gamma_{2}}{D^{2}}, \\
8 N^{3}-\frac{8 \Gamma_{1}}{D} N^{2}+\frac{2}{D^{2}}\left(\Gamma_{2}+\Gamma_{1}^{2}\right) N+\frac{\Gamma_{3}-\Gamma_{1} \Gamma_{2}}{D^{3}}=0 .
\end{gathered}
$$

The positive root of the cubic polynomial equation, Eq. (17), is given by

$$
\begin{gathered}
N=\frac{\Gamma_{7}}{D}=\frac{1}{D}\left(\Gamma_{5}+\Gamma_{6}+\frac{\Gamma_{1}}{3}\right), \\
\Gamma_{4}=\left(\frac{3 \Gamma_{2}-\Gamma_{1}^{2}}{36}\right)^{3}+\left(\frac{\Gamma_{1}^{3}}{216}+\frac{\Gamma_{3}}{16}-\frac{\Gamma_{1} \Gamma_{2}}{48}\right)^{2}, \\
\Gamma_{5}=\left(-\frac{\Gamma_{1}^{3}}{216}-\frac{\Gamma_{3}}{16}+\frac{\Gamma_{1} \Gamma_{2}}{48}+\sqrt{\Gamma_{4}}\right)^{1 / 3}, \\
\Gamma_{6}=\left(-\frac{\Gamma_{1}^{3}}{216}-\frac{\Gamma_{3}}{16}+\frac{\Gamma_{1} \Gamma_{2}}{48}-\sqrt{\Gamma_{4}}\right)^{1 / 3} .
\end{gathered}
$$

The expression for the relative wavelength is obtained with the aid of Eqs. (16) and (18a) as follows:

$$
\frac{L}{H_{c}}=\frac{\pi\left(\sqrt{1+8 F_{0}^{2}}-1\right)}{F_{0}^{2 / 3} \sqrt{3 \Gamma_{7}^{2}-2 \Gamma_{1} \Gamma_{7}+\Gamma_{2}}},
$$

where $L$ is the wavelength [L]; $F_{0}$ (dimensionless) is the Froude number of the approach flow; and $H_{c}$ is the critical flow depth [L]. Using the boundary conditions $H=H_{J 1}$ and $H_{x}=0$ at the toe of the jump, the constants $C_{1}$ and $C_{2}$ are easily determined. For positive real values of these constants, the relative wave height can be computed by the following equation:

$$
\frac{\varepsilon}{H_{c}}=\frac{2\left(3-\sqrt{1+8 F_{0}^{2}}\right) \exp \left(-N C_{2}\right)}{F_{0}^{2 / 3}\left(2-k^{2} C_{2}^{2}\right)},
$$

where $\varepsilon$ is the vertical distance between the crest and trough of the wave (wave height) [L]. Equations (19) and (20) are obtained by using the Bélanger (1845) equation based on a nearly horizontal channel approximation.

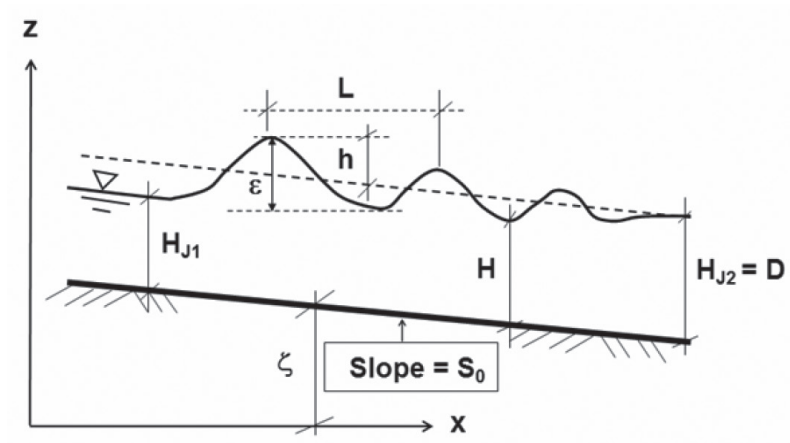

Fig. 2 Undular open-channel flows in a prismatic channel with a uniform approach flow

\section{NUMERICAL SCHEME}

Because of its non-linear nature, a closed-form solution cannot be obtained for Eq. (10a). This equation includes a third-order spatial derivative term; hence, it requires an accurate third- or higher-order numerical scheme for its solution (Abbott et al., 1978). In this study, a fourth-order finite-difference scheme developed by Bashforth and Adams (1883) is employed to solve the model equation numerically. Using this scheme, the flow depths at an unknown time, $t_{3}$, are com- 
puted from the flow parameters at the known time levels, $t_{0}, t_{1}$ and $t_{2}$, using an iterative technique. After eliminating the $\partial Q / \partial x$ term by using Eq. (11), Eq. (10a) becomes

$$
\begin{gathered}
\vartheta \frac{\partial H}{\partial t}=\Omega, \\
\Omega=\frac{\partial Q}{\partial t}+\frac{\sigma_{2}}{15} \frac{Q^{2}}{B} H_{x x x}+\frac{\sigma_{1}}{6} \frac{Q^{2}}{A} \zeta_{x} H_{x x}+\left(g A-\beta \frac{Q^{2} B}{A^{2}}\right) H_{x} \\
+\frac{Q^{2}}{A}\left(\frac{\sigma_{1}}{6} \zeta_{x x x} H+\frac{\sigma_{3}}{3} \zeta_{x x} \zeta_{x}\right)+g A \zeta_{x}+\frac{f Q^{2} P_{w}}{8 A^{2}}\left(1+\zeta_{x}^{2}\right), \\
\vartheta=\left(\beta \frac{2}{H}+\frac{\sigma_{1}}{3} \zeta_{x x}+\frac{2 \sigma_{2}}{15} H_{x x}\right) Q .
\end{gathered}
$$

The numerical scheme applied comprises the steps of a predictor and a corrector. In the predictor step, the third-order Adams-Bashforth scheme (Bashforth and Adams, 1883) is used to approximate the time derivative appearing in Eq. (21a). The resulting discretized equation can be written as

$$
H_{j}^{P}=H_{j}^{n}+\frac{\Delta t}{12}\left(\frac{23 \Omega_{j}^{n}}{\vartheta_{j}^{n}}-\frac{16 \Omega_{j}^{n-1}}{\vartheta_{j}^{n-1}}+\frac{5 \Omega_{j}^{n-2}}{\vartheta_{j}^{n-2}}\right),
$$

where the superscript $P$ (dimensionless) stands for the predicted values at different nodal points; the superscripts $n, n-1$ and $n-2$ (dimensionless) denote the condition at the known time levels; $j$ (dimensionless) denotes the computational point; and $\Delta t$ is the time step [T] In the step of a predictor, $\Omega_{j}$ is determined by using the values of the flow parameters at the known time levels in Eq. (21b).

In the corrector step, the time integration is performed by applying the fourth-order Adams-Moulton scheme using the predicted and known values of the parameters. For this purpose, Eq. (21a) is discretized by using the scheme equation originally proposed by Adams (Bashforth and Adams, 1883), and the resulting expression becomes

$$
H_{j}^{c}=H_{j}^{n}+\frac{\Delta t}{24}\left(\frac{9 \Omega_{j}^{P}}{\vartheta_{j}^{P}}+\frac{19 \Omega_{j}^{n}}{\vartheta_{j}^{n}}-\frac{5 \Omega_{j}^{n-1}}{\vartheta_{j}^{n-1}}+\frac{\Omega_{j}^{n-2}}{\vartheta_{j}^{n-2}}\right),
$$

where the superscript $c$ (dimensionless) refers to the value of the parameter after the corrector step. For this computational step, the value of $\Omega_{j}^{P}$ is determined by utilizing the estimated values of the parameters. Because of the strong non-linear coefficients associated with the flow equation, an iterative procedure is applied to obtain the final solutions of the problem at the corrector step. The convergence of the numerical solutions is assessed on the basis of the relative change in the solution criterion with a convergence tolerance of $10^{-4}$.

Equations (22) and (23) are applied at the computational nodes between $j=1$ and $j=m-1$ to model the unsteady, transitional open-channel flow problems. The nodal values at $j=0$ and $m$ are determined from the specified boundary conditions, which will be described in detail in the next section. The first, second, and third spatial partial derivative terms, which appear in Eq. (21b), are discretized by using the finite-difference approximations as follows (Bickley, 1941):

$$
\begin{gathered}
H_{x, j}=\frac{1}{12 \Delta x}\left(-H_{j+2}+8 H_{j+1}-8 H_{j-1}+H_{j-2}\right), \\
H_{x x, j}=\frac{1}{(\Delta x)^{2}}\left(H_{j-1}-2 H_{j}+H_{j+1}\right), \\
H_{x x x, j}=\frac{1}{(\Delta x)^{3}}\left(-H_{j-2}+3 H_{j-1}-3 H_{j}+H_{j+1}\right)
\end{gathered}
$$

The above equations are replaced by forward and backward finite-difference approximations for the nodes near the upstream and downstream ends of the computational domain. The time-varying discharge, appearing in Eq. (21b), is also discretized by using the thirdand fourth-order accurate finite-difference equations (Bickley, 1941) for the predictor and corrector steps, respectively.

\section{NUMERICAL EXPERIMENTS}

In this section, the applicability of the proposed model is assessed by simulating three different types of hydraulic jumps in open channels. These jumps are the: (i) free hydraulic jump; (ii) undular hydraulic jump with an aspect ratio, $B / H_{J 1}\left(H_{J 1}\right.$ is the supercritical sequent depth [L]), ranging from 2.6 to 10.4 ; and (iii) submerged hydraulic jump with different degrees of submergence. These steady, curvilinear transitional flow problems were solved herein as unsteady flow problems, by using time as an iteration parameter till the final steady-state solutions were reached. For accurately modeling the mean flow characteristics of the transitional flow problems, a finer step size, which is less than $15 \%$ of the flow depth at the outflow section, was used. For all the test cases, a time step in the order 0.01 $\mathrm{s}$ was employed.

As for the initial condition, the flow at $t=0$ was assumed to be a supercritical uniform flow with a flow depth equal to the depth specified at the inflow section. The slope of the free surface, which was computed by a gradually-varied flow equation, was specified at this section for the free and undular hydraulic jumps and at the outflow section for the submerged jump. A constant subcritical flow depth, which could force the jump to occur, was also specified at the outflow section. All the specified boundary conditions remained unchanged during the numerical computations.

The values of the velocity distribution parameter, $\omega$, were estimated using the experimental data of Hager and Hutter (1983), Long (1991), and Chanson (1995) for the aforementioned flow problems. For the free and submerged hydraulic jumps, the $\omega$ values of 2.0 and 3.7 , respectively, provided a fairly good approximation for the distribution of the velocity in the recirculation zone. In the case of an undular hydraulic jump with $F_{0} \leq 1.72$, the effect of a surface roller on the horizontal velocity is insignificant. Hence, a value of $\omega$ equal to 0.75 was found to be satisfactory for this test case. These values were used in the numerical model to simulate the hydraulic jumps by treating the turbulent flow problems as single-phase flow problems.

\subsection{Free hydraulic jumps}

The results of the model for the turbulent hydraulic jump were verified using the experimental data of Gharangik (1988). These experiments were performed in a straight rectangular $14 \mathrm{~m}$ long, 460 $\mathrm{mm}$ wide, and $915 \mathrm{~mm}$ deep flume. The flume had metal walls and glass-walled sections. The free-surface profiles in the section of the flume with metal walls were measured at equi-spaced intervals by a point gage with an accuracy of $0.30 \mathrm{~mm}$. For the approaching Froude numbers ranging from 2.3 to 7.0 , the rectangular grids on the glasswalled section were used to measure the free-surface profiles of the jump and its locations. Full details of the description of the experimental system can be found in Gharangik (1988).

Figure 3 displays the normalized free-surface profiles $\left(\eta / H_{c}\right.$ versus $x / H_{c}$ ) of the free jumps with the approaching Froude numbers $2.30,4.23,5.74$, and 6.65 . As can be seen, the jump length and its overall free-surface profile were predicted reasonably well by the proposed model. In addition, the location of the toe of the jump was predicted fairly well. In the subcritical flow region near the end of the 

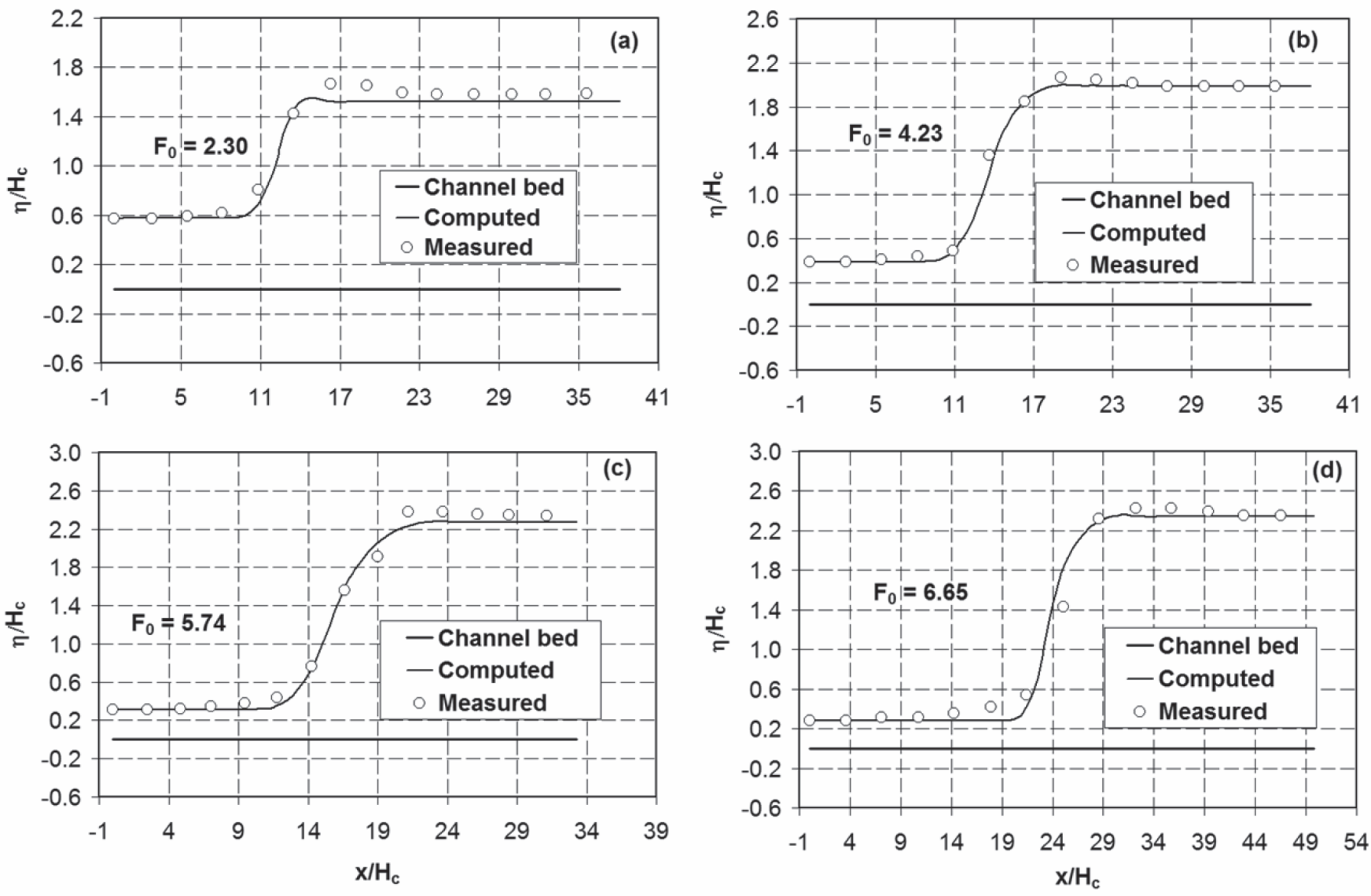

Fig. 3 Normalized free-surface profiles for free hydraulic jumps with different Froude numbers of the approach flow

jump, however, the elevations of the free surface were slightly underestimated. This discrepancy is due to the flow bulking effects caused by air entrainment in the experiments (Resch et al., 1974; Takahashi and Ohtsu, 2017), which have not been accounted for by the present model. A short distance further downstream, the agreement between the numerical results and the experimental data was satisfactory for all the Froude numbers tested. The results of this test case attested the model's capability and provided valuable insight into the approximate 2D flow structure of the free hydraulic jump.

\subsection{Undular hydraulic jumps}

An undular hydraulic jump was considered to test the validity of the numerical model. As described by Chanson and Montes (1995), for an approaching Froude number less than 1.20 to 1.25, the flow features of the undular jump resemble the nature of a curvilinear open-channel flow with substantial streamline vertical curvatures. Such a 2D transitional flow character allows us to develop suitable scenarios for assessing the effects of the vertical acceleration on the performance of the model. The experimental data of Chanson (1995) and Gotoh et al. (2005) were used to validate the numerical results. These experiments were carried out in smooth, rectangular cross-section channels with fully developed upstream shear flow conditions. In both experiments, the flow depths along the channel centerline were measured using a point gage. In addition, a Pitot tube was employed by Chanson (1995) to measure the velocity and pressure distributions.

Figure 4 depicts the normalized free-surface profiles of the undular hydraulic jumps for the cases of $F_{0} \leq 1.25$ and $F_{0}>1.25$. As shown in Fig. 4a and c, the predictions of the maximum and minimum free-surface elevations at the wave crests and troughs agreed closely with the experimental data. However, the results of the comparison presented in Fig. $4 \mathrm{~b}$ and d showed some notable deviations of the numerical results from the experimental data, especially in the flow region downstream of the second wave $\left(x / H_{c}>18\right)$. This discrepancy may be attributed to the 3D characteristics of the undular flow caused by the appearance of cross-channel shock waves as $F_{0}$ exceeds the limiting value of 1.25 (Reinauer and Hager, 1995; Chanson and Montes, 1995). As a depth-averaged Boussinesq-type model, it does not accurately portray plane open-channel flows with cross-waves. Overall, the model efficiently captured the effects of the streamline curvature of the moderately curved transitional flow.

The characteristics of the first wave of the undular jump problems were numerically analyzed, and the results were compared with the experimental data and the results of the approximate equation proposed by Mandrup-Andersen (1978). Fig. 5a shows the variation of the relative wave height, $\varepsilon / H_{c}$, with the Froude number of the approach flow. Similar to the solution of the approximate Eq. (20), the results of Eq. (10a) for the relative wave height showed a good correlation with the experimental data. As depicted in Fig. 5b, the computed relative wavelengths, $L / H_{c}$, agreed reasonably well with the measurements. However, the simplifying assumptions used to develop Eq. (19) significantly affected its solution. As expected, the relative wavelength decreases when the Froude number of the approach flow increases.

Figure $5 \mathrm{c}$ and $\mathrm{d}$ presents the pressure distributions at the crest and trough of the first wave for an undular flow with $F_{0}$ equal to 1.25 . In this figure, the non-dimensional pressure, $p / p_{0}$, is plotted as a function of $\lambda$, in which $p_{0}$ is the hydrostatic pressure at the bed $\left[\mathrm{ML}^{-1} \mathrm{~T}^{-2}\right]$. It is evident from Fig. $5 \mathrm{c}$ and $\mathrm{d}$ that the dynamic bed pressure due to the effect of the streamline curvature is about $9 \%$ of the corresponding static pressure. For the near-critical open-channel flow problem considered here, the proposed model accurately described the 2D struc- 

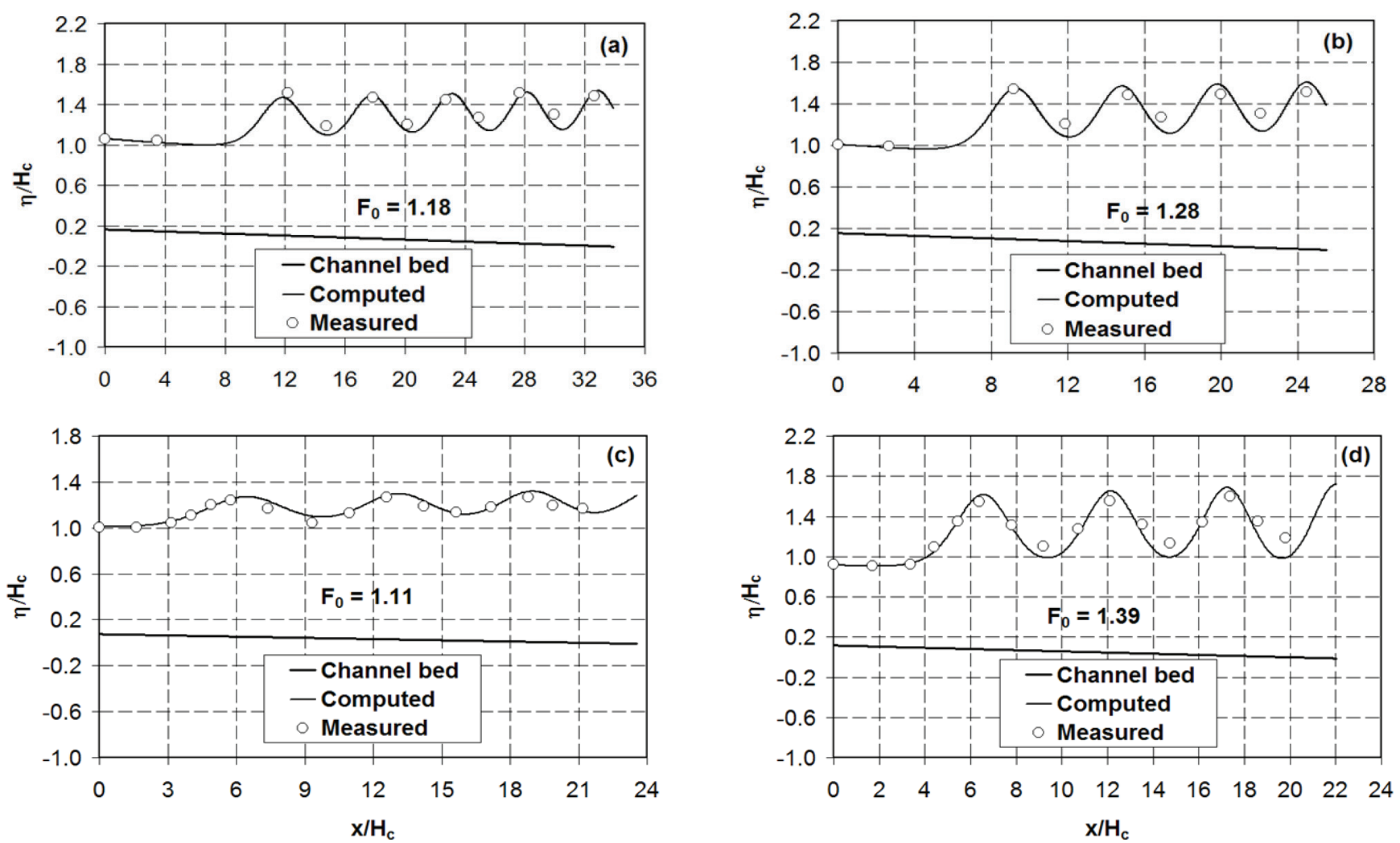

Fig. 4 Normalized free-surface profiles for near-critical open-channel flows in mild-slope channels. The numerical results are compared with the experimental data of Chanson (1995) in (a) and (b) and with Gotoh et al. (2005) in (c) and (d)
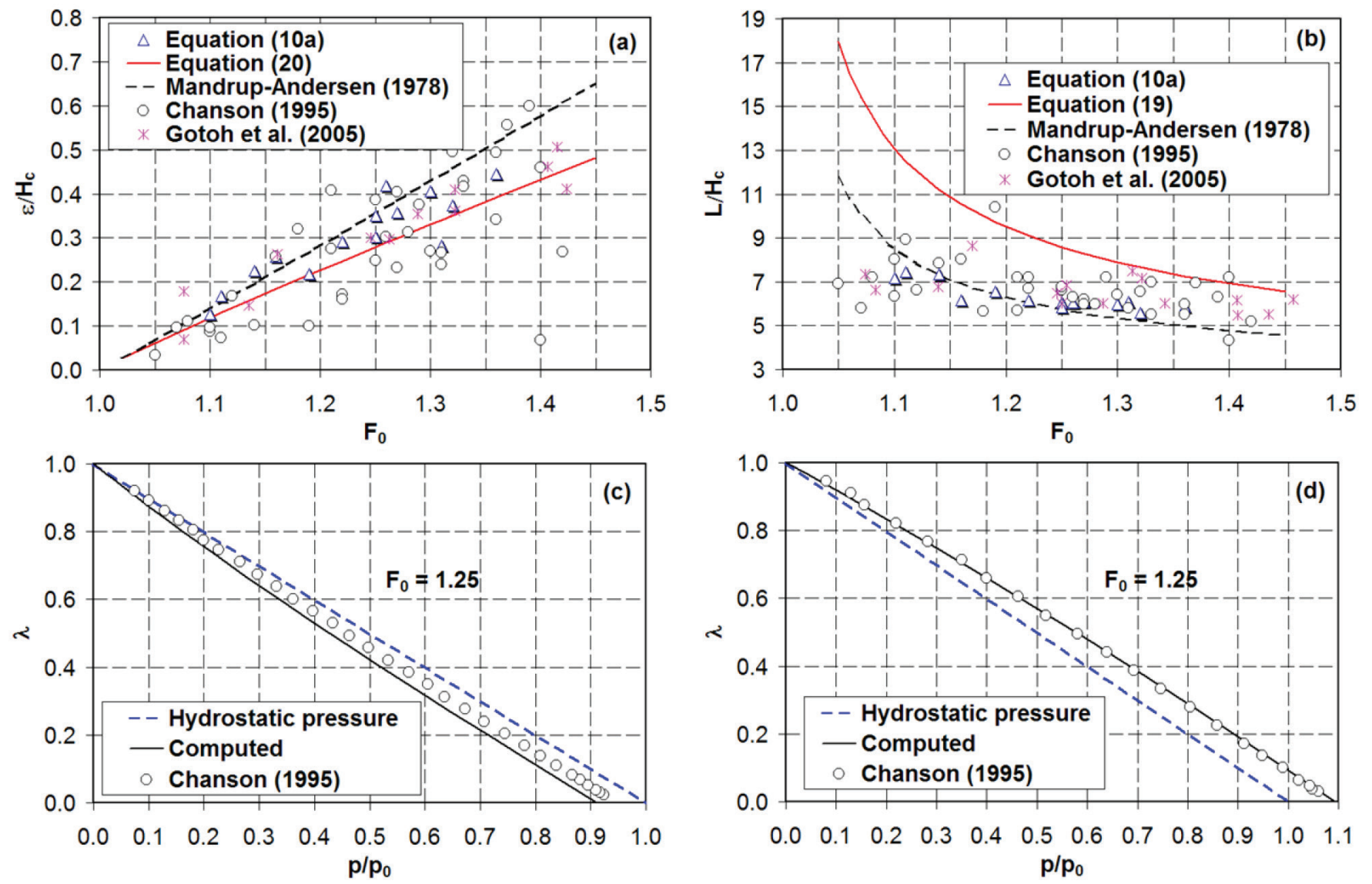

Fig. 5 Characteristics of the first wave of an undular jump: (a) relative wave height; (b) relative wavelength; (c) pressure distribution at the wave crest; and (d) pressure distribution at the wave trough 
ture of the curvilinear flow field; hence, its overall performance for predicting the mean flow characteristics was satisfactory.

\subsection{Submerged hydraulic jumps}

Long (1991) conducted a series of laboratory experiments on submerged hydraulic jumps in a $467 \mathrm{~mm}$ wide, $515 \mathrm{~mm}$ deep, and 7.5 $\mathrm{m}$ long horizontal rectangular channel with vertical glass walls and an aluminum bed. The experiments covered ten conditions with the submergence ratio, $S\left(=\left[H_{\mathrm{m}}-H_{J 2}\right] / H_{J 2}\right)$, where $H_{J 2}$ is the subcritical sequent depth [L]), varying from 22 to $170 \%$ and the approaching Froude numbers ranging from 3.0 to 8.2 . The free-surface profiles and the velocity distributions at different sections were measured using a point gage and Laser Doppler Anemometry (LDA), respectively. The accuracy of the flow depth measurement in a calm water condition was about $\pm 0.5 \mathrm{~mm}$. The experimental data of selected runs, which are listed in Tab. 1, were used to investigate the validity of the numerical results. Further details of the experimental system can be found in Long (1991).

For the problem considered, the flow depth, which was specified as a boundary condition at the upstream end, was computed using the following equation (Chow, 1959):

$$
H_{0}=H_{m}\left(1+2 F_{m}^{2}\left(1-\frac{H_{m}}{H_{G}}\right)\right)^{1 / 2}
$$

where $H_{0}$ and $H_{\mathrm{m}}$ are the flow depths at the upstream and downstream ends [L], respectively; $H_{G}$ is the opening height of the sluice gate [L]; and $F_{\mathrm{m}}$ (dimensionless) is the Froude number of the downstream quasi-uniform flow. Fig. 6 shows a comparison of the predicted free-surface profiles with the measurements. As can be seen, the free-surface elevations were predicted reasonably well by the model except in the flow region near the middle of the recirculation zone. In this region, the influence of the 3D characteristics of the flow was significant. As a result, the model overestimated the free-surface elevations especially for flows with a lower submergence ratio and a higher Froude number of the approach flow (see Fig. 6b). The agreement between the numerical and experimental results was much better for test cases having a higher submergence ratio and a lower Froude number of the approach flow. In the flow region where the $3 \mathrm{D}$ effects are insignificant $\left(x / H_{c}>15\right)$, the results of the model showed close agreement with the experimental data. In general, the prediction of the streamwise locations of the minimum free-surface elevation was satisfactory.

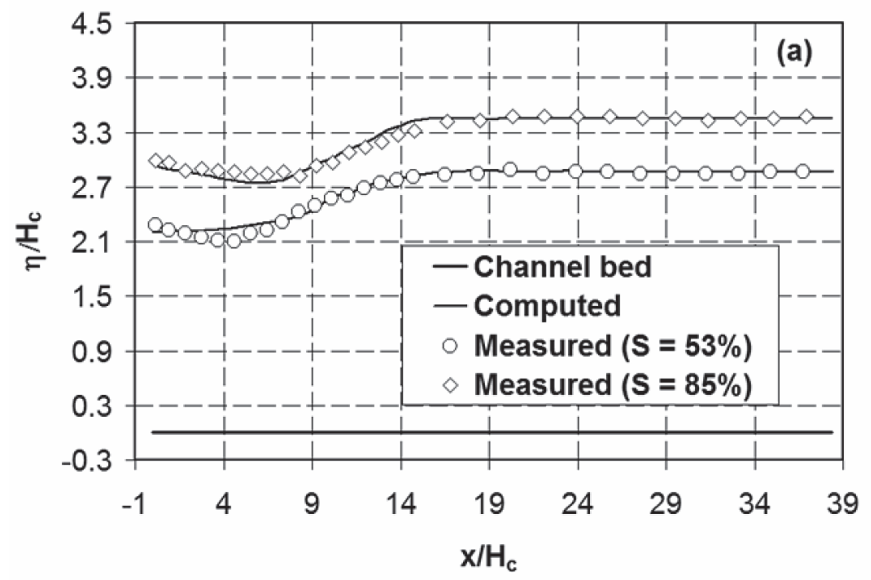

Tab. 1 Values of the parameters for the selected experimental runs

\begin{tabular}{|c|c|c|c|}
\hline Run & $\mathbf{H}_{\mathbf{G}}(\mathbf{m m})$ & $\mathbf{F}_{\mathbf{0}}$ & $\mathbf{S}(\mathbf{\%})$ \\
\hline $\mathrm{T} 2$ & 25 & 3.20 & 53 \\
\hline $\mathrm{T} 3$ & 25 & 3.19 & 85 \\
\hline $\mathrm{T} 6$ & 25 & 5.49 & 63 \\
\hline $\mathrm{T} 8$ & 15 & 8.19 & 24 \\
\hline
\end{tabular}

The preceding numerical experiments demonstrated that the mean flow characteristics of the turbulent open-channel flows were satisfactorily simulated by the proposed depth-averaged Boussinesq-type model. This simple and computationally efficient model provided vital information regarding the streamwise variation of the flow depth, wavelength, amplitude, and length of the hydraulic jump. In practice, such information can be used to develop or refine a conceptual design of an energy dissipator or a prismatic canal that conveys undular open-channel flows. During the iterative design process, the design modifications of a hydraulic jump energy dissipator can easily be incorporated in the present model by changing only a few parameters. In the case of a complex curvilinear transitional flow with varying hydraulic characteristics across the channel width, the detailed design can be done by implementing a 3D numerical model.

\section{CONCLUDING REMARKS}

A numerical model, which ignores the turbulence characteristics of the flow, was developed and used to investigate the salient features of curvilinear open-channel flows as well as the dependence of the characteristics of such flows on the Froude number of the approach flow and the curvature of the streamline. The present approach applied a dynamic pressure correction so that the effects of the non-uniform velocity and non-hydrostatic pressure distributions were implicitly incorporated into the model. The Adams-Bashforth-Moulton numerical scheme was used to discretize and solve the model equation. The applicability of the model was then assessed by conducting numerical experiments related to transitional open-channel flows from a supercritical to a subcritical state such as free and submerged hydraulic jumps and an undular jump. The results of the model for the free-surface profile, pressure distribution, and characteristics of the first wave of an undular jump were compared with those measured in the laboratory flumes.

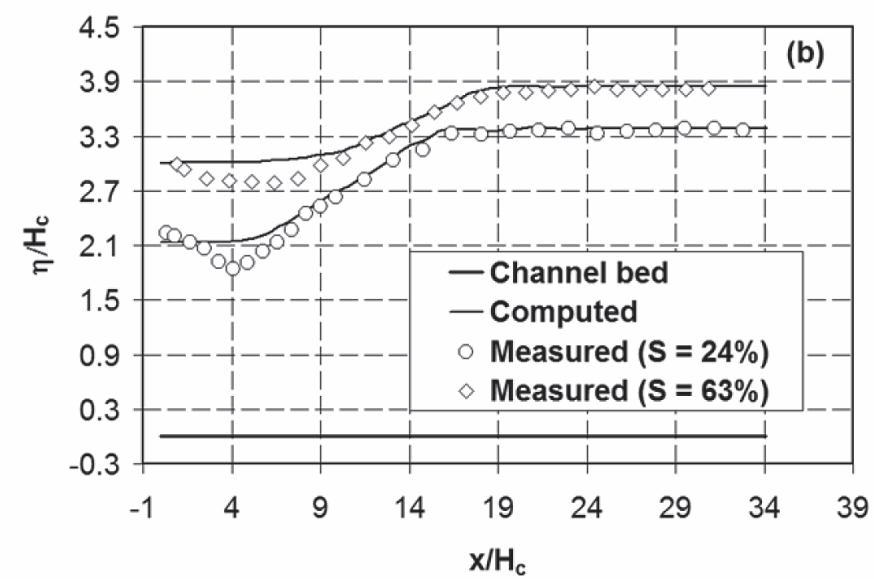

Fig. 6 Normalized free-surface profiles for submerged hydraulic jumps with different degrees of submergence 
The conclusions of this study may be stated as follows:

1. The numerical results for the free and submerged hydraulic jumps showed a good correlation with the experimental data except in the flow regions, where the effects of the 3D characteristics of the flow and the bulking of flow caused by air entrainment are substantial. Overall, the results of the comparison suggested the validity of the proposed model for predicting the plane-flow characteristics of such types of rapidly-varied open-channel flows and most importantly, from the standpoint of an energy dissipator design, the length and profile of the hydraulic jumps.

2. For the case of an undular hydraulic jump with $F_{0} \leq 1.25$, the $2 \mathrm{D}$ characteristics of the moderately curved transitional flow were accurately portrayed by the proposed depth-averaged Boussinesq-type model. However, the appearance of cross-channel shock waves at higher Froude numbers affected the overall quality of the results of the model. The proposed model was able to satisfactorily predict the wavelength and wave height of the jump, thereby demonstrating its capacity for handling the 2D undular flow problems. The results of this numerical experiment confirmed that the applicability of the model is limited to such types of near-critical open-channel flows without cross-channel shock waves.

The results of the above investigation clearly establish the credentials of the proposed model for simulating the mean flow characteristics of transitional open-channel flows with curved streamlines. Considering its simplicity and computational efficiency, the present model can be most effectively used as an engineering tool to refine designs prior to laboratory-scale modeling, thereby expediting the iterative design process and drastically reducing both the time and costs associated with the testing of a laboratory-scale model.

\section{REFERENCES}

Abbott, M. B. - Rodenhuis, G. S. (1972) A numerical simulation of the undular hydraulic jump. J. Hydraul. Res., 10(3), 239-257.

Abbott, M. B. - Petersen, H. M. - Skovgaard, O. (1978) On the numerical modeling of short waves in shallow water. J. Hydraul. Res., 16(3), 173-204.

Bakhmeteff, B. A. - Matzke, A. E. (1936) The hydraulic jump in terms of dynamic similarity. Trans. ASCE, 101(1), 630-647.

Bashforth, F. - Adams, J. C. (1883) An Attempt to Test the Theories of Capillary Action by Comparing the Theoretical and Measured Forms of Drops of Fluid, with an Explanation of the Method of Integration Employed in Constructing the Tables which Give the Theoretical Forms of Such Drops. Cambridge University Press, Cambridge, UK, 18-19. Available online: https://www.archive. org/details/attempttest00bashrich (accessed on 04 May 2020).

Bayon-Barrachina, A. - Lopez-Jimenez, P. A. (2015) Numerical analysis of hydraulic jumps using OpenFOAM. J. Hydroinform., $17,662-678$.

Bélanger, J. B. (1845) Notes sur l'Hydraulique (Notes on Hydraulic Engineering). École Royale des Ponts et Chaussées, Paris, France, Session 1845-1846, 84-85 [in French]. Available online: https:// patrimoine.enpc.fr/document/ENPC02_COU_4 2381_1845 (accessed on 10 July 2020).

Benjamin, T. B. - Lighthill, M. J. (1954) On cnoidal waves and bores. Proc. Roy. Soc. Lond. A, 224(1159), doi:10.1098/ rspa.1954.0172.

Bickley, W. G. (1941) Formulae for numerical differentiation. Math. Gaz., 25(263), 19-27, doi:10.2307/3606475.

Bose, S. - Castro-Orgaz, O. - Dey, S. (2012) Free-surface profiles of undular hydraulic jumps. J. Hydraul. Eng., 138(4), 362-366.

Boussinesq, J. (1877) Essai Sur la Théorie des Eaux Courantes (Essay on the Theory of Water Flow). Mémoires Présentés par Divers Savants à l'Académie des Sciences, Paris, 23(1), 196-198 [in French].

Chanson H. (1995) Flow Characteristics of Undular Hydraulic Jumps: Comparison with Near-Critical Flows. Research Report CH45/95. Department of Civil Engineering, University of Queensland, Brisbane, Australia.
Chanson, H. - Montes, J. S. (1995) Characteristics of undular hydraulic jumps. Experimental apparatus and flow patterns. J. Hydraul. Eng., 121(2), 129-144.

Chanson, H. (2000) Boundary shear stress measurements in undular flows: Application to standing wave bed forms. Water Resour. Res., 36(10), 3063-3076.

Chanson H. (2009) Current knowledge in hydraulic jumps and related phenomena. A survey of experimental results. Eur. J. Mech. B/ Fluids, 28(2), 191-210.

Chow, V. T. (1959) Open-Channel Hydraulics. McGraw-Hill, New York, NY, USA, 60.

Dey, S. - Sarkar, A. (2008) Characteristics of turbulent flow in submerged jumps on rough beds. J. Eng. Mech., 134(1), doi:10.1061/ (ASCE)0733-9399(2008)134:1(49).

Fawer, C. (1937) Etude de Quelques Écoulements Permanents à Filets Courbes (Study of Some Permanent Flows with Curved Filaments). Docteur ès Sciences Techniques Thèse, Université de Lausanne, Lausanne, Switzerland [in French], doi:10.5075/ epfl-thesis-9.

Fenton, J. D. - Zerihun, Y. T. (2007) A Boussinesq approximation for open-channel flow. In: Proceedings of the 32nd Congress, IAHR, Venice, Italy, 2-6 July, CD-ROM.

Gharangik, A. M. (1988) Numerical Simulation of Hydraulic Jump. Master's Thesis, Washington State University, Pullman, WA, USA.

Grillhofer, W. - Schneider, W. (2003) The undular hydraulic jump in turbulent open-channel flow at large Reynolds numbers. Phys. Fluids, 15(3), 730-735.

Gotoh, H. - Yasuda, Y. - Ohtsu, I. (2005) Effect of channel slope on flow characteristics of undular hydraulic jumps. WIT Trans. Ecol. Environ., 83, 33-43.

Govinda Rao, N. S. - Rajaratnam, N. (1963) The submerged hydraulic jump. J. Hydraul. Div., 89(HY1), 139-162.

Hager, W. H. - Hutter, K. (1983) Approximate treatment of the plane hydraulic jump with separation zone above the flow zone. J. Hydraul. Res., 21(3), 195-204. 
Hager, W. H. - Hutter, K. (1984) On pseudo-uniform flow in open-channel hydraulics. Acta Mech., 53, 183-200.

Hager, W. H. (1993) Classical hydraulic jump: Free-surface profiles. Can. J. Civ. Eng., 20(3), 536-539.

Khan, A. A. - Steffler, P. M. (1996) Physically based hydraulic jump model for depth-averaged computation. J. Hydraul. Eng., 122, 540-548.

Liu, M. - Rajaratnam, N. - Zhu, D. (2004) Turbulence structure of hydraulic jumps of low Froude numbers. J. Hydraul. Eng., 130, 511-520.

Long, D. (1991) An Experimental Investigation and $\kappa-\varepsilon$ Turbulence Modeling of Submerged Hydraulic Jumps. Ph.D. Thesis, Department of Civil Engineering, University of Alberta, Edmonton, AL, Canada.

Long, D. - Steffler, P. M. - Rajaratnam, N. (1991) A numerical study of submerged hydraulic jumps. J. Hydraul. Res., 29(3), 293-308.

Ma, F. - Hou, Y. - Prinos, P. (2001) Numerical calculation of submerged hydraulic jumps. J. Hydraul. Res., 39(5), 493-503.

Madsen, P. A. - Simonsen, H. J. - Pan, C. H. (2005) Numerical simulation of tidal bores and hydraulic jumps. Coast. Eng., 52, 409-433.

Mandrup-Andersen, V. (1978) Undular hydraulic jump. J. Hydraul. Div., 104(HY8), 1185-1188.

McCorquodale, J. A. - Khalifa, A. (1983) Internal flow in hydraulic jumps. J. Hydraul. Eng., 109(5), doi:10.1061/(ASCE)07339429(1983)109:5(684).

Mignot, E. - Cienfuegos, R. (2010) Energy dissipation and turbulent production in weak hydraulic jumps. J. Hydraul. Eng., 136, doi:10.1061/(ASCE)HY.1943-7900.0000124.

Montes, J. S. (1986) A study of the undular jump profile. In: Proceedings of the 9th Australasian Fluid Mechanics Conference, Auckland, New Zealand, 8-12 Dec., 148-151.

Montes, J. S. - Chanson, H. (1998) Characteristics of undular hydraulic jumps. Experiments and analysis. J. Hydraul. Eng., 124(2), 192-205.

Ohtsu, I. - Yasuda, Y. - Gotoh, H. (2001) Hydraulic condition for undular-jump formations. J. Hydraul. Res., 39(2), 203-209.

Padulano, R. - et al. (2017) Hydraulic design of a USBR type II stilling basin. J. Irrig. Drain. Eng., 143(5), doi:10.1061/(ASCE) IR.1943-4774.0001150.

Qingchao, L. - Drewes, U. (1994) Turbulence characteristics in free and forced hydraulic jumps. J. Hydraul. Res., 32(6), 877-898.
Rajaratnam, N. - Subramanya, K. (1968) Profiles of the hydraulic jump. J. Hydraul. Div., 94(HY3), 663-674.

Reinauer, R. - Hager, W. H. (1995) Non-breaking undular hydraulic jump. J. Hydraul. Res., 33(5), 683-698.

Resch, F. J. - Leutheusser, H. J. (1972a) Reynolds stress measurements in hydraulic jumps. J. Hydraul. Res., 10(4), 409-429.

Resch, F. J. - Leutheusser, H. J. (1972b) Le ressaut hydraulique: Mesure de turbulence dans la région diphasique (The hydraulic jump: Turbulence measurements in the two-phase flow region). La Houille Blanche, 4, 279-293 [in French].

Resch, F. J. - Leutheusser, H. J. - Alemu, S. (1974) Bubbly twophase flow in hydraulic jump. J. Hydraul. Div., 100(HY1), $137-$ 149.

Richard, G. L. - Gavrilyuk, S. L. (2013) The classical hydraulic jump in a model of shear shallow-water flows. J. Fluid Mech., $725,492-521$.

Ryabenko, A. A. (1990) Conditions favorable to the existence of an undulating jump. Hydrotech. Constr., 24(12), doi:10.1007/ BF01434602.

Takahashi, M. - Ohtsu, I. (2017) Effects of inflows on air entrainment in hydraulic jumps below a gate. J. Hydraul. Res., 55(2), 259-268.

Witt, A. - Gulliver, J. - Shen, L. (2018) Numerical investigation of vorticity and bubble clustering in an air entraining hydraulic jump. Comput. Fluids, 172, 162-180.

Zerihun, Y. T. (2008) Numerical modeling of open-channel flow with dual free surfaces - Free overfall. In: Proceedings of the 8th International Conference on Hydro-Science and -Engineering, Nagoya, Japan, 8-12 Sept., CD-ROM.

Zerihun, Y. T. (2016) Modeling free-surface flow with curvilinear streamlines by a non-hydrostatic model. J. Hydrol. Hydromech., 64(3), 281-288.

Zerihun, Y. T. (2017a) A numerical study of non-hydrostatic shallow flows in open channels. Arch. Hydro-Eng. Environ. Mech., 64(1), $17-35$.

Zerihun, Y. T. (2017b) A non-hydrostatic depth-averaged model for hydraulically steep free-surface flows. Fluids, 2(4), doi:10.3390/ fluids2040049.

Zigrang, D. J. - Sylvester, N. D. (1982) Explicit approximations to the solution of Colebrook's friction factor equation. AIChE J., 28(3), 514-515. 